Monika Roth Sigrist ${ }^{a}$

Barbara Schoop ${ }^{a}$

Nicole Möslia

Regierungsrätin Heidi Hanselmann eröffnete das Fachsymposium Gesundheit mit der Frage: «Integrative Medizin (k)ein neues Thema?» Die Politikerin hob hervor, dass die Zeichen der Zeit in der Gesundheitsversorgung erkannt worden sind und eine Balance zwischen der traditionellen und integrativen Medizin und Pflege gefunden werden soll.

Die thematische Einführung übernahmen Dr. med. Marc Schläppi vom Kantonsspital St. Gallen und Prof. Dr. med. Peter Heusser von der Universität Witten-Herdecke. Wichtige Begriffsklärungen und Gründe für die Beanspruchung von Komplementärmedizin waren aus den Vorträgen ebenso zu hören wie die Tatsache, dass Integrative Medizin zu einem akademischen Entwicklungsgebiet geworden ist, das intensive Forschung betreibt.

Entwicklung der Arzneimittel zwischen Volksmedizin und Schulmedizin

Prof. Dr. pharm. François Ledermann (Abb. 1), Pharmaziehistoriker am Institut für Medizingeschichte der Universität Bern, zeigte in seinem Vortrag die wichtigsten Meilensteine auf, welche die Entwicklung von der Volksmedizin zur Schulmedizin prägten. Maja dal Cero (Abb. 2) vom Institut für systematische Botanik der Universität Zürich ermöglichte einen Einblick in die Tradition der Arzneipflanzenanwendung und stellte

*Kantonsspital St. Gallen, Schweiz

\title{
Fortbildung
}

Schweiz Z Ganzheitsmed 2012;24:218-220

DOI: 10.1159/000341324

\section{Fachsymposium Gesundheit «Integrative Medizin und Pflege - mehr als ein Trend» in St. Gallen}

«Integrative Medizin und Pflege - mehr als ein Trend» - mit diesem Titel präsentierte sich das 5. Fachsymposium Gesundheit am 18. und 19. Januar 2012 in St. Gallen. Der Veranstalter, das Kantonsspital St. Gallen, erachtet die Ergänzung der konventionellen Medizin durch die Integrative Medizin und Pflege als zukunftsweisend für eine ganzheitliche Behandlung. Der Anlass bot mit seinen Referaten einen breiten und facettenreichen Überblick über die Integrative Medizin und Pflege. Insbesondere wurde über die Wirksamkeit, Zweckmässigkeit und Wirtschaftlichkeit sowie die aktuelle politische Debatte gesprochen.

ihre ethnobotanische Studie zu den Kräuterkundigen in der Schweiz vor. In ihrem Referat kam sie zu der interessanten Schlussfolgerung, dass im Verlauf der Geschichte eine Vielfalt von pflanzlichen Arzneimitteln in der Schweiz genutzt wurde, sich diese aber aktuell auf einen kleinen Prozentsatz reduziert hat, und dass unabhängig von der jeweiligen medizinischen Kultur eine hohe Gemeinsamkeit in der Nutzung von pflanzlichen Arzneimitteln besteht.

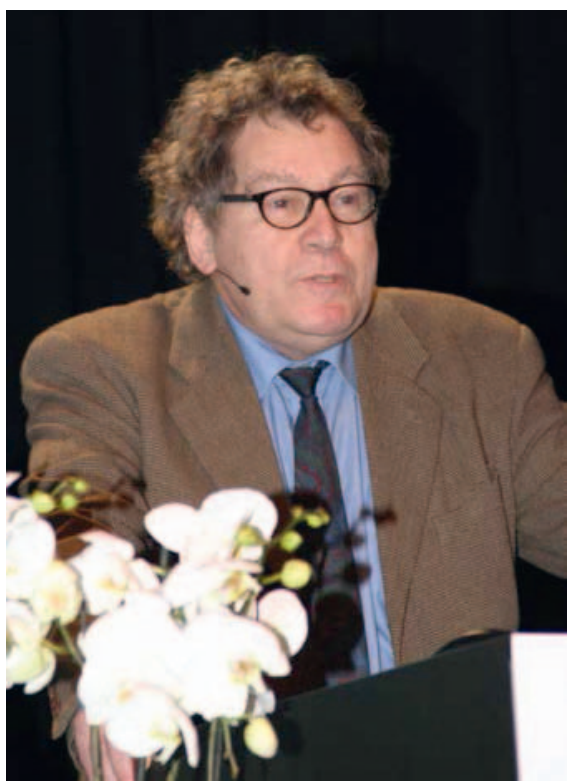

Abb. 1.

Prof. Dr. pharm. François Ledermann.
Forschung zur komplementären und integrativen Medizin Standortbestimmung und Wege für die Zukunft

Prof. Dr. med. Claudia Witt (Abb. 3) erläuterte in ihrem methodischen Exkurs die Probleme der randomisierten kontrollierten Studien (RCTs) in der medizinischen Forschung und reflektierte kritisch den Wert von Placebo-Studien. Sie plädierte für «Comparative Effectiveness Re-

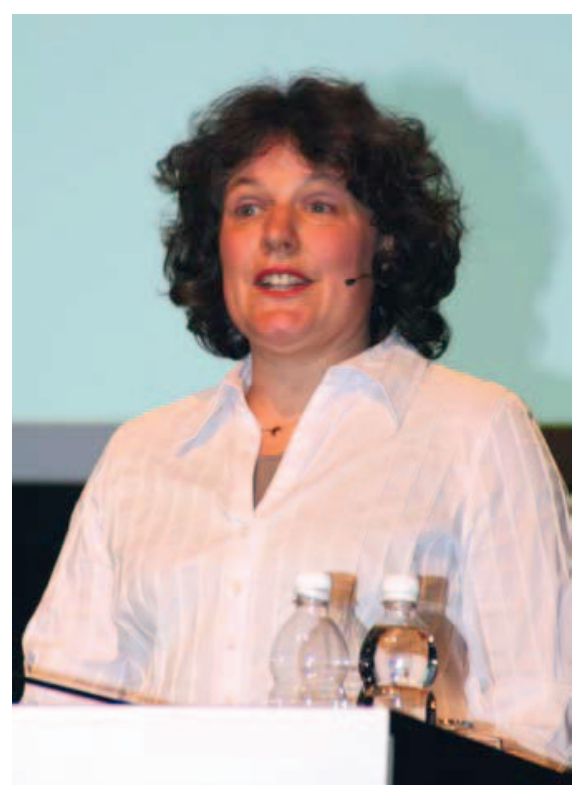

Abb. 2.

Maja dal Cero.
(๑) 2012 S. Karger GmbH, Freiburg
Monika Roth Sigris

Departement Pflege, Kantonsspital St. Gallen

9007 St. Gallen, Schweiz

Tel. +41 714941276

monika.roth@kssg.ch 
search» (CER) zur Erzeugung und Synthese von Evidenz, die den Nutzen und das Risiko alternativer Methoden zur Prävention, Diagnostik, Behandlung und/oder Steuerung einer Erkrankung vergleicht. Ziel der CER ist es, Verbraucher, Ärzte, Versicherer und politische Entscheidungsträger darin zu unterstützen, informierte Entscheidungen $\mathrm{zu}$ treffen, welche die Gesundheitsversorgung auf der individuellen und gesellschaftlichen Ebene verbessern.

Als kritische Stimme in der Veranstaltung stellte Michael Rüegg, lic. phil., in einem philosophischen Exkurs den von einzelnen Richtungen der Komplementärmedizin implizierten Anspruch an ein ganzheitliches Menschenbild infrage. Die Entwicklung einer Gesamtsicht eines Menschen, so Rüegg, gehöre in die Philosophie und in die Religion und nicht in eine empirische Wissenschaft wie die Medizin. In der Medizin müssten Wissen und Weltanschauung getrennt werden. Findet diese Trennung in der Behandlung von Klienten nicht statt, so laufe die Komplementärmedizin Gefahr, dass Glaube praktiziert, aber als Wissenschaft angepriesen werde.

Integrative Medizin und Pflege in der praktischen Versorgung

Neben den theoretisch ausgerichteten Referaten fokussierten verschiedene Vorträge auf die patientenzentrierte Versorgung und veranschaulichten anhand von Beispielen, wo sich die Integrative Medizin und Pflege in Ergänzung zur sogenannten Schulmedizin etablieren konnte.

So im Spital Scuol im Unterengadin, das seit 2007 mit dem Projekt des ganzheitlichen Heilens Integrative Medizin anbietet. Dr. med Hannes Graf, leitender Arzt der Abteilung für Komplementär- und Palliative Medizin, erläuterte, dass es möglich ist, die Komplementärmedizin in einem öffentlichen Spital zu integrieren. Elke Rey-Burg berichtete als Stationsleite-

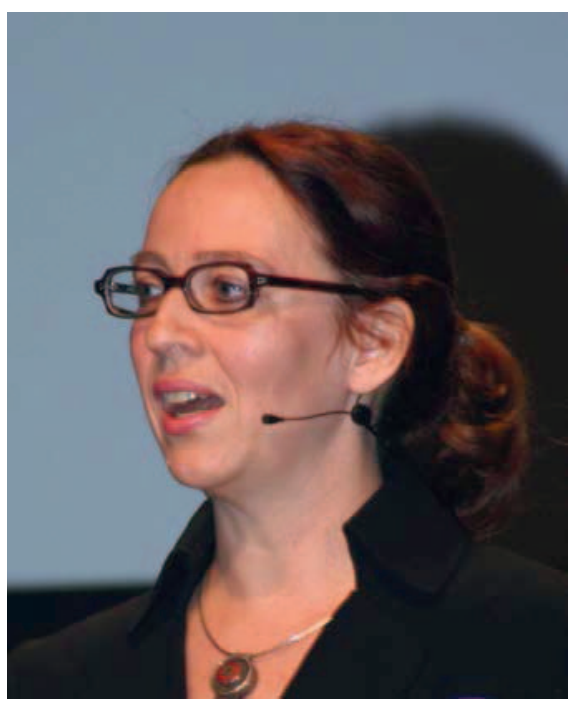

Abb. 3. Prof. Dr. med. Claudia Witt.

rin einer «Palliative Care»-Bettenstation am Kantonsspital St. Gallen von ihren Erfahrungen mit der Einführung der Integrativen Medizin und über deren positive Auswirkungen auf die Pflegepraxis. Prof. Dr. med. Gustav Dobos aus der Klinik EssenMitte bot in seinen Ausführungen einen vertieften Einblick in die verschiedenen komplementärmedizinischen Angebote. Er ging ausführlich auf die Arbeit der Mind-Body-Therapeuten mit ihren Angeboten, wie beispielsweise Meditation (Mindfulness-Based Stress Reduction/MBSR), Achtsamkeit und Qigong, zur «Relaxation Response» ein. GuaSha- und Schröpfkopf-Massagen sind Anwendungungen, die von speziell dafür ausgebildeten Pflegefachpersonen durchgeführt werden. In einem Film kamen Patienten zu Wort, die ihre Geschichte und ihre positiven Erfahrungen mit diesen Heilmethoden erzählten.

Bei allen Praxisprojekten wurde betont, dass für eine erfolgreiche Umsetzung von Integrativer Medizin und Pflege nicht die Summe der ausgewählten komplementärmedizinischen Angebote im Vordergrund steht, sondern dass auf eine gut funktionierende Zusammenarbeit zwischen allen Beteiligten, wie Ärzteschaft, Pflegefachpersonen und

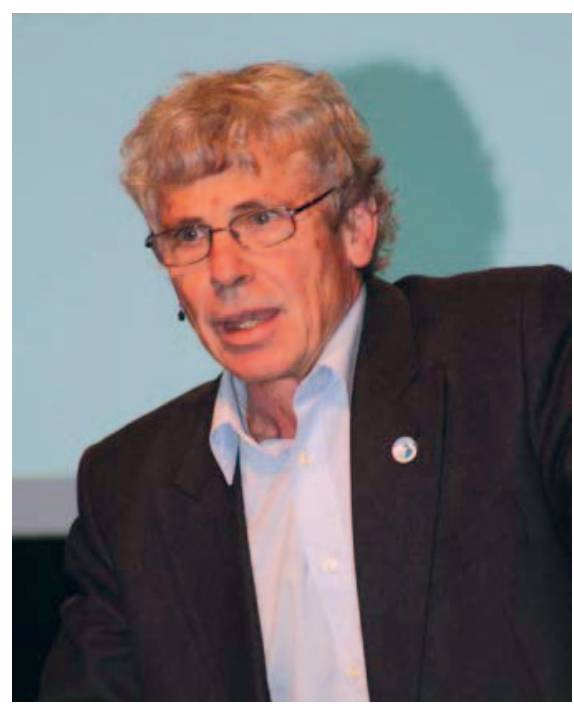

Abb. 4. Dr. med. Hansueli Albonico.

Therapeuten, grosser Wert gelegt werden muss.

\section{Politische Debatte}

In den Referaten von Dr. med. Hansueli Albonico (Präsident UNION schweizerischer komplementärmedizinischer Ärzteorganisationen) (Abb. 4) und Dr. med. Yvonne Gilli (Nationalrätin) wurde deutlich, welch intensive politische Bemühungen auch nach der gewonnenen Volksabstimmung «Ja zur Komplementärmedizin» nötig sind, damit im Heilmittelgesetz die Kernforderungen der Initiative (Heilmittelschatz bewahren, Berufsanerkennung und Qualitätssicherung der nichtärztlichen Therapeuten, Aufnahme der fünf Methoden in die Grundversicherung sowie das Fördern von Lehre und Forschung) verankert werden.

\section{Workshops}

Im Workshop Basale Stimulation ${ }^{\circledR}$ wurden die Grundlagen des Konzeptes anhand einer Fallgeschichte praxisorientiert erläutert und diskutiert. Den Teilnehmenden wurde die Gelegenheit geboten, sich selbst auf ein Angebot zur somatischen Wahr- 
nehmung einzulassen, was zu einem regen Austausch über das Erlebte anregte. Nach einem kurzen Input zum Anwendungs- und Betriebskonzept Basale Stimulation am Kantonsspital St. Gallen konnten unterschiedliche wahrnehmungsfördernde Positionierungen geübt werden.

Wickel und Kompressen in der Pflege können durch den gezielten Einsatz von Temperatur, Feuchtigkeit und Substanzen Beschwerden lindern und die Lebensqualität steigern. In diesem Workshop wurden die Teilnehmenden in die Wirkungsweisen und Techniken der Ölkompressen eingeführt. Die dazugehörigen Materialien und eine Auswahl von Ölen (Gemische aus Fetten und ätherischen Ölen) wurden vorgestellt. In einer praktischen Sequenz bot sich die Gelegenheit, Ölkompressen aneinander anzuwenden und deren entspannende und wohltuende Wirkung selbst zu erleben.

\section{Fazit}

Dank der Vielseitigkeit hervorragender Referate und Diskussionen auf hohem Niveau konnte ein breites Fachpublikum erfahren, dass die Integrative Medizin und Pflege in der Tat mehr als ein Trend ist. Weitere Informationen finden sich unter www.fachsymposium.ch. 